\section{LUPUS SCIENCE\& MEDICINE}

\title{
Investigations into SCAMP5, a candidate lupus risk gene expressed in plasmacytoid dendritic cells
}

\author{
Mustafa H Ghanem (1) , ${ }^{1,2}$ Andrew J Shih (1) , ${ }^{1}$ Himanshu Vashistha (1) , \\ Latanya N Coke (D) , ${ }^{1}$ Wentian Li (D) , ${ }^{1}$ Sun Jung Kim (D) , ${ }^{1,2}$ \\ Kim R Simpfendorfer (D) ,1,2 Peter K Gregersen (D) 1,2
}

To cite: Ghanem MH, Shih AJ, Vashistha $\mathrm{H}$, et al. Investigations into SCAMP5, a candidate lupus risk gene expressed in plasmacytoid dendritic cells. Lupus Science \& Medicine 2021;8:e000567. doi:10.1136/ lupus-2021-000567

- Additional supplemental material is published online only. To view, please visit the journal online (http://dx.doi.org/10. 1136/lupus-2021-000567).

KRS and PKG are joint senior authors.

Received 25 August 2021 Accepted 1 October 2021

\section{Check for updates}

(c) Author(s) (or their employer(s)) 2022. Re-use permitted under CC BY-NC. No commercial re-use. See rights and permissions. Published by BMJ.

${ }^{1}$ The Institute of Molecular Medicine, Northwell Health Feinstein Institutes for Medical Research, Manhasset, New York, USA

${ }^{2}$ Donald and Barbara Zucker School of Medicine at Hofstra/ Northwell, Hempstead, New York, USA

Correspondence to Dr Peter K Gregersen; pgregers@northwell.edu and Dr Kim R Simpfendorfer; Ksimpfendo@northwell.edu

\section{ABSTRACT}

Objective We have investigated the molecular function of SCAMP5, a candidate risk gene for SLE exclusively expressed in plasmacytoid dendritic cells (pDCs) among peripheral leucocytes.

Methods We tested the independence of the association in SCAMP5 with SLE by performing conditional analyses. We profiled the expression pattern of SCAMP5 among circulating leucocytes at the transcript and protein levels. Using lentiviral vectors, we localised the subcellular distribution of SCAMP5 alongside the interferon secretory pathway. We analysed pDCs for the expression of SCAMP5 and interferon production capacity by SCAMP5 genotype. Finally, we examined pDC-specific SCAMP5 isoforms by total RNAseq analysis and examined for genotypeassociated quantitative differences therein.

Results A conditional analysis revealed evidence of an independent genetic association of SCAMP5 with SLE. Among circulating leucocytes, SCAMP5 is uniquely expressed in pDCs at the transcript and protein levels, with main presence in the Golgi apparatus and minor presence at the cell periphery. In live cells, SCAMP5 displayed dynamic Golgi-cell surface trafficking and localised with the interferon secretory pathway. SCAMP5 did not differ in expression levels in pDCs between genotyped donors; however, a transient interferon secretory defect was noted in pDCs from donors carrying the risk genotype.

Conclusions SCAMP5 constitutes a novel SLE risk gene on the basis of genomic data and expression in a cell type widely implicated in SLE pathogenesis. While we could not find evidence of quantitative expression differences in SCAMP5 between genotyped donors, SCAMP5 remains an attractive gene to explore given its highly restricted expression pattern and colocalisation with interferon secretion.

\section{INTRODUCTION}

Plasmacytoid dendritic cells (pDCs) are rare innate immune cells which express the pattern recognition receptors toll-like receptors 7 and 9 (TLR7 and TLR9), which together with their constitutive expression of the type I interferon (IFN-I) inducer interferon regulatory factor 7 (IRF7) are poised to rapidly respond to viral infection. ${ }^{1}$ Secretion

\begin{aligned} \hline KEY MESSAGES \\ \hline WHAT IS ALREADY KNOWN ABOUT THIS SUBJECT? \\ \hline$\Rightarrow$ SLE is associated with overactivity of the type I in- \\ terferon pathway, and plasmacytoid dendritic cells \\ (pDCs) are major producers of type I interferon in this \\ disease. \\ WHAT DOES THIS STUDY ADD? \\ $\Rightarrow$ A polymorphism on chromosome 15q24 associates \\ with SLE by virtue of a novel candidate SLE risk \\ gene, SCAMP5. \\ $\Rightarrow$ We Confirmed that among circulating leucocytes, \\ SCAMP5 is uniquely expressed in pDCs at the tran- \\ script and protein levels, with main presence in \\ the Golgi apparatus and minor presence at the cell \\ periphery. \\ $\Rightarrow$ We detected a transient interferon secretory defect \\ in pDCs from donors homozygous for the SLE risk \\ genotype, providing a potential link between a func- \\ tional consequence of the genetic risk variant and \\ the SLE risk association. \\ HOW MIGHT THIS IMPACT ON CLINICAL PRACTICE \\ OR FUTURE DEVELOPMENTS? \\ $\Rightarrow$ Molecular dissection of heritable traits in type I in- \\ terferon secretion may unveil novel opportunities for \\ drug development. \end{aligned}

of massive amounts of IFN-I, particularly IFN- $\alpha$, is characteristic of pDCs, and downstream signalling in response to IFN drives induction of an antiviral programme in host cells, an 'IFN signature' that is constitutively activated in the blood of many patients with SLE. ${ }^{2}$

A role of pDCs in the pathogenesis of SLE has also been suggested by their presence in cutaneous lupus lesions and in lupus nephritis. ${ }^{34}$ Furthermore, antibody-mediated depletion of pDCs has demonstrated therapeutic efficacy in human trials. ${ }^{5}$ From the genomic standpoint, several SLE risk genes are enriched for expression in pDCs, including TLR7, IRF7, IRF8 and DNASE1L $3 .{ }^{67}$ 
pDC production of IFN-I is potentially a major driver of the IFN signature. IFN-I signalling has been shown to enhance antigen presentation, promote $\mathrm{B}$ cell differentiation and suppress regulatory $\mathrm{T}$ cells-processes central to the development of SLE. ${ }^{8-10}$

In their recent meta-analysis of genomewide association studies(GWAS) of SLE, Bentham et $a l^{11}$ reported an association at single-nucleotide polymorphism (SNP) rs2289583, with an OR of 1.19 and a p value of $6 \times 10^{-15}$. rs2289583 occurs in intron 6 of SCAMP5, a member of the secretory carrier membrane protein (SCAMP) family. The SCAMPs are tetraspan membrane proteins with cytoplasmic N-termini and C-termini and both luminal and extraluminal peptide loops connecting the transmembrane domains along the surface of intracellular trafficking vesicles (online supplemental figure S1). ${ }^{12}$ SCAMPs 1-3 are ubiquitously expressed, whereas SCAMPs 4-5, lacking the characteristic N-terminal NPF repeats of the other SCAMPs, display a more limited expression pattern, largely restricted to the brain. ${ }^{13}$ This expression profile of SCAMP5 in the central nervous system appears to be conserved among mammalian species.

Outside of the brain, we noted a strong signal for SCAMP5 expression in pDCs. ${ }^{14}$ Given the multiplicity of evidence for the role played by pDCs in SLE, and the association of this risk locus with the disease, we sought to determine whether SCAMP5 contributed to SLE by modulating a key function of pDC biology: IFN-I secretion.

\section{METHODS}

See online supplemental material.

\section{RESULTS}

\section{SCAMP5 associates independently with SLE on chr15q24}

A recent SLE GWAS meta-analysis reported on susceptibility loci concentrated in genes involved in innate and adaptive immune function. ${ }^{11}$ This study coupled analyses of their respective discovery and replication cohorts with a previous SLE GWAS from our group. ${ }^{15}$ Among the associations was the polymorphism rs2289583. CSK was annotated as the likely gene explaining the association signal, citing prior work from our group establishing a link between CSK and SLE in this genomic region. ${ }^{16}$

However, inspection of SNP associations across this region reveals that the lead SNP from this analysis lies within the SCAMP5 gene, $\sim 155 \mathrm{~kb}$ telomeric to CSK. This raised the question as to whether CSK drives the association signal at rs2289583. We examined the primary data in this region, with individual-level data on 4036 SLE cases and 6959 controls from the main GWAS of the aforementioned study. ${ }^{11}$ As shown in figure 1A (left panel), two SNPs associated with SLE risk achieve genome-wide significance in the region: rs2289583 in the last intron of SCAMP5 $\left(\mathrm{OR}=1.2, \mathrm{p}=3.4 \times 10^{-9}\right)$ and $\mathrm{rs} 1378940$ in the first intron of CSK $\left(\mathrm{OR}=1.17, \mathrm{p}=2.8 \times 10^{-8}\right)$. Our group previously identified $\mathrm{B}$ cell phenotypes associated with a lupus risk variant in the CSK gene (rs34933034) ${ }^{16}$ The rs34933034 SNP was not genotyped in the Bentham et al data set. In fact, this variant in CSK is poorly imputed by other variants in the regionthe lead SNP in CSK in this analysis (rs1378940) exhibits an $r^{2}=0.34$ with $r$ s34933034. Moreover, the signal at $r s 2289583$ is unlikely to reflect the effects of our previously identified association at CSK, as the linkage disequilibrium (LD) between these two SNPs is also quite modest $\left(\mathrm{r}^{2}=0.2\right.$ between rs34933034 and rs2289583).

Therefore, we carried out formal conditional analysis to seek evidence for SCAMP5 as a secondary or alternative driver of the SLE risk association in the $C S K / S$ CAMP5 region. On conditioning on the associated SNP within SCAMP5, the signal at rs 1378940 within CSK is substantially reduced (figure 1A, middle panel). In contrast, the association signal in SCAMP5 (rs2289583 $\mathrm{OR}=1.2, \mathrm{p}=3.4 \times 10^{-9}$ ) was only partially reduced following conditioning on the lead risk-association SNP in CSK $\left(\mathrm{p}_{\text {cond }}=1.66 \times 10^{-4}\right)$ (figure $1 \mathrm{~A}$, right panel), indicating that the signal at SCAMP5 is independent of the association signals observed within CSK itself.

\section{SCAMP5 is uniquely expressed in pDCs among peripheral blood leucocytes}

Having established that SCAMP5 may represent a novel SLE risk gene, we sought to probe the functional biology underlying this association. SCAMP5 is reported to be expressed uniquely by pDCs among peripheral blood leucocytes in humans (BioGPS ${ }^{17}$ and Human Protein Atlas ${ }^{14}$; online supplemental figure S2). To confirm this, we sorted leucocyte subsets from four healthy donors and examined SCAMP5 expression by reverse transcription (RT)-PCR. SCAMP5 transcript was detected in pDCs from all donors and was absent in their B, T and NK cells, as well as monocytes and conventional dendritic cells (cDCs) (figure 1B, left panel). To determine whether SCAMP5 is expressed at the protein level in pDCs, we resolved lysates from several leucocyte subsets and detected SCAMP5 protein using the polyclonal antibody $(\mathrm{pAb})$ against SCAMP5 employed by the Human Protein Atlas. We found that SCAMP5 is indeed expressed at the protein level only in pDCs (figure 1B, middle panel). To agnostically determine whether any other (unsorted) peripheral blood leucocyte subset expressed SCAMP5, RBC-depleted whole blood was stained intracellularly for SCAMP5 using the same pAb. Only pDCs stained for SCAMP5, confirming the unique expression of this protein in the circulation (figure 1B, right panel).

To localise expression of SCAMP5 within pDCs, fixed and permeabilised pDCs were stained for SCAMP5 and visualised by immunofluorescence. Colocalisation was evident with the Golgi marker golgin-97, with additional evidence of staining for SCAMP5 at the cell periphery (figure 1C).

The novelty of SCAMP5 as an SLE risk gene, coupled with the striking expression pattern restricted to $\mathrm{pDCs}$, a cell type highly invoked in SLE pathogenesis, prompted us to further explore a role of SCAMP5 in modulating pDC function. 
A

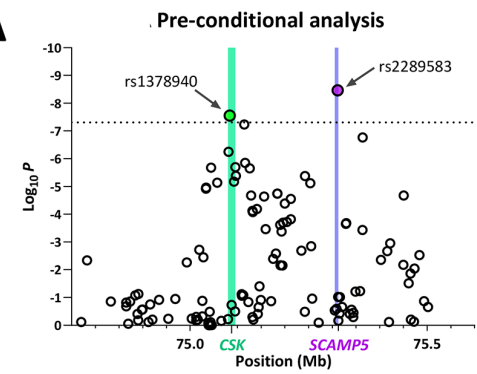

Conditioned on rs2289583 in SCAMP5

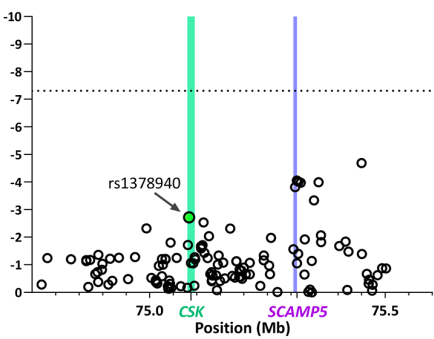

Conditioned on rs1378940 in CSK

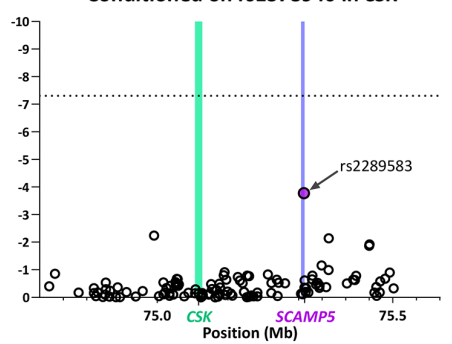

B

$\underline{\text { RT-PCR }}$

Western Blot

Flow Cytometry
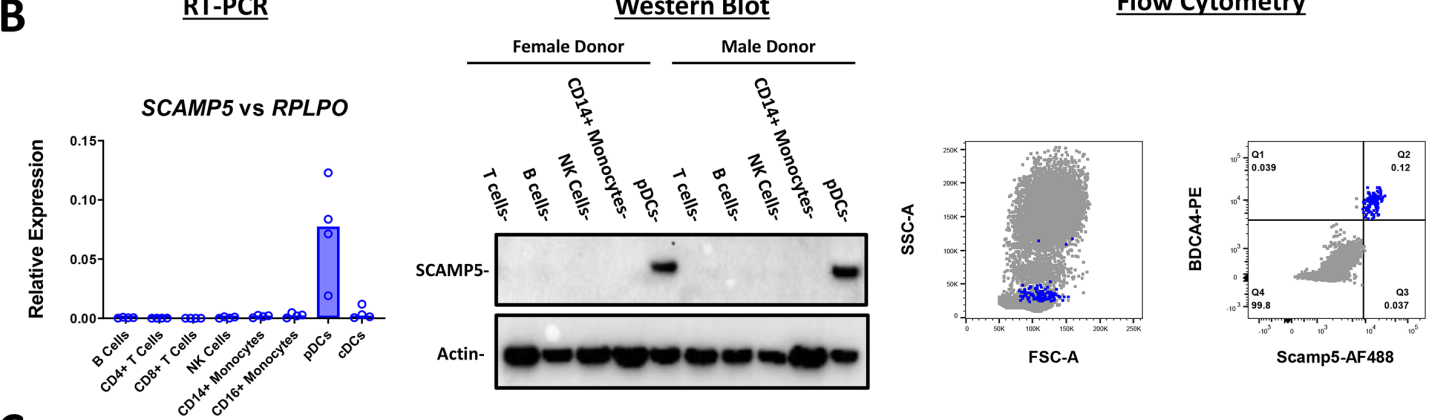

C

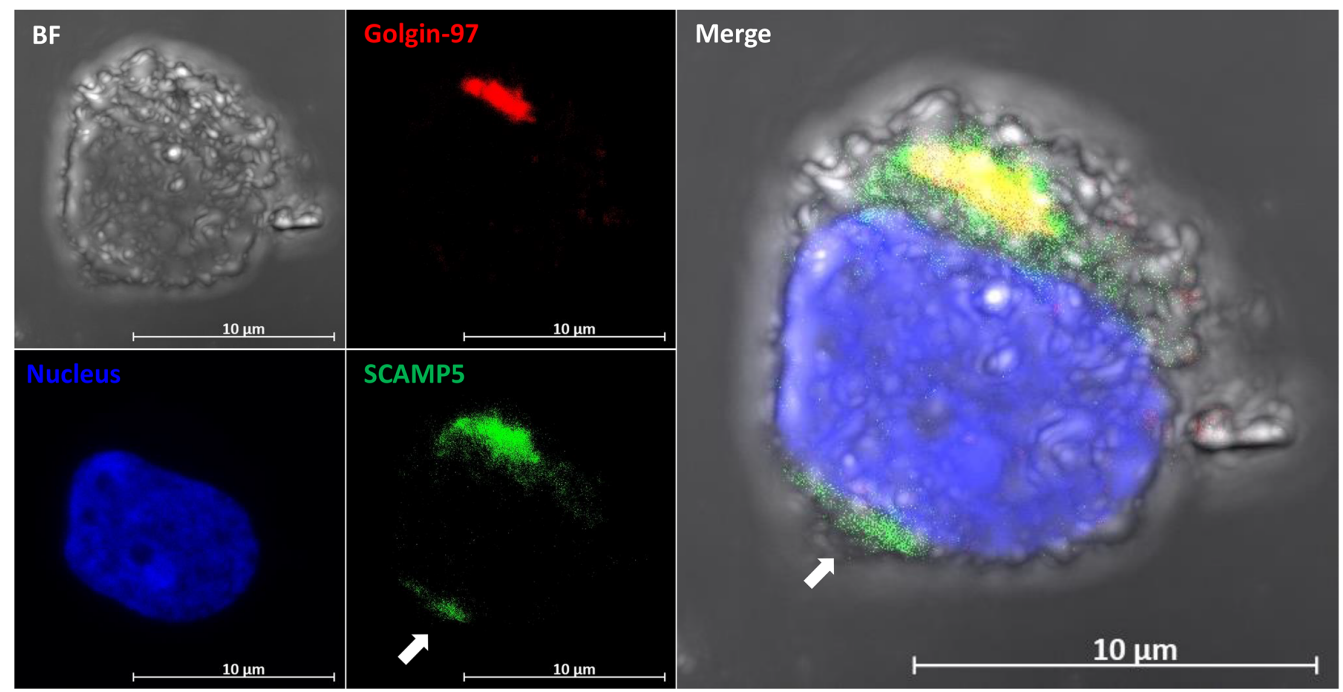

Figure 1 SCAMP5 associates independently with SLE and is expressed in the Golgi apparatus and cell periphery of pDCs. (A) Genomic coordinates and conditional analyses on lead SLE association SNPs in SCAMP5 and CSK. (Left) Summary of SNP associations in the CSK-SCAMP5 region on chromosome 15 from Bentham et al ${ }^{11}$ for 4036 SLE cases and 6959 controls. The association values following conditioning on the lead risk-association SNP in SCAMP5 (rs2289583 in purple) (middle) or the lead risk-association SNP in CSK (rs1378940 in green) (right). Genome-wide significance at $5 \times 10^{-8}$ is indicated by the dotted line. Vertical shading depicts the location of the SCAMP5 gene (purple) and CSK gene (green). Positions are from the 2009 (GRCh37/ hg19) assembly. (B) SCAMP5 is expressed exclusively in pDCs among human circulating leucocytes. (Left) Quantitative RT-PCR for SCAMP5 reveals significant expression in pDCs and not in lymphocytes, monocytes or cDCs. Each data point represents one donor's flow sorted cells. (Middle) Likewise, SCAMP5 protein is detected by a specific antibody in pDCs and not in other immune cells in western blot. (Right) Intracellular labelling for SCAMP5 in RBC-depleted human whole blood analysed by flow cytometry indicates that SCAMP5 is present in the small population of BDCA4hi cells (pDCs) and absent in all other cells in the circulation, including the granulocyte population as reflected by light scatter. (C) Subcellular immunolocalisation of SCAMP5 in pDCs. Representative confocal Z-stack maximal projection image of a PDC stained for SCAMP5 and a Golgiresident membrane protein, golgin-97. SCAMP5 concentrates in the Golgi, with minor presence at the cell periphery (arrow). $63 \times$ magnification with oil immersion. Staining with DAPI delineates the nucleus. cDCs, conventional dendritic cells; pDCs, plasmacytoid dendritic cells; RT-PCR, reverse transcription PCR.

SCAMP5 traffics between the Golgi stack and cell surface and colocalises with clathrin and the IFN-I export pathway

The SCAMP protein family remains understudied. To develop tools to enable a more complete understanding of SCAMP5 in particular, several SCAMP5 constructs were generated and cloned into lentiviral vectors. To localise and track SCAMP5 in live cells, a SCAMP5-mKate2 fusion protein was designed. SCAMP5-mKate2 was transduced 

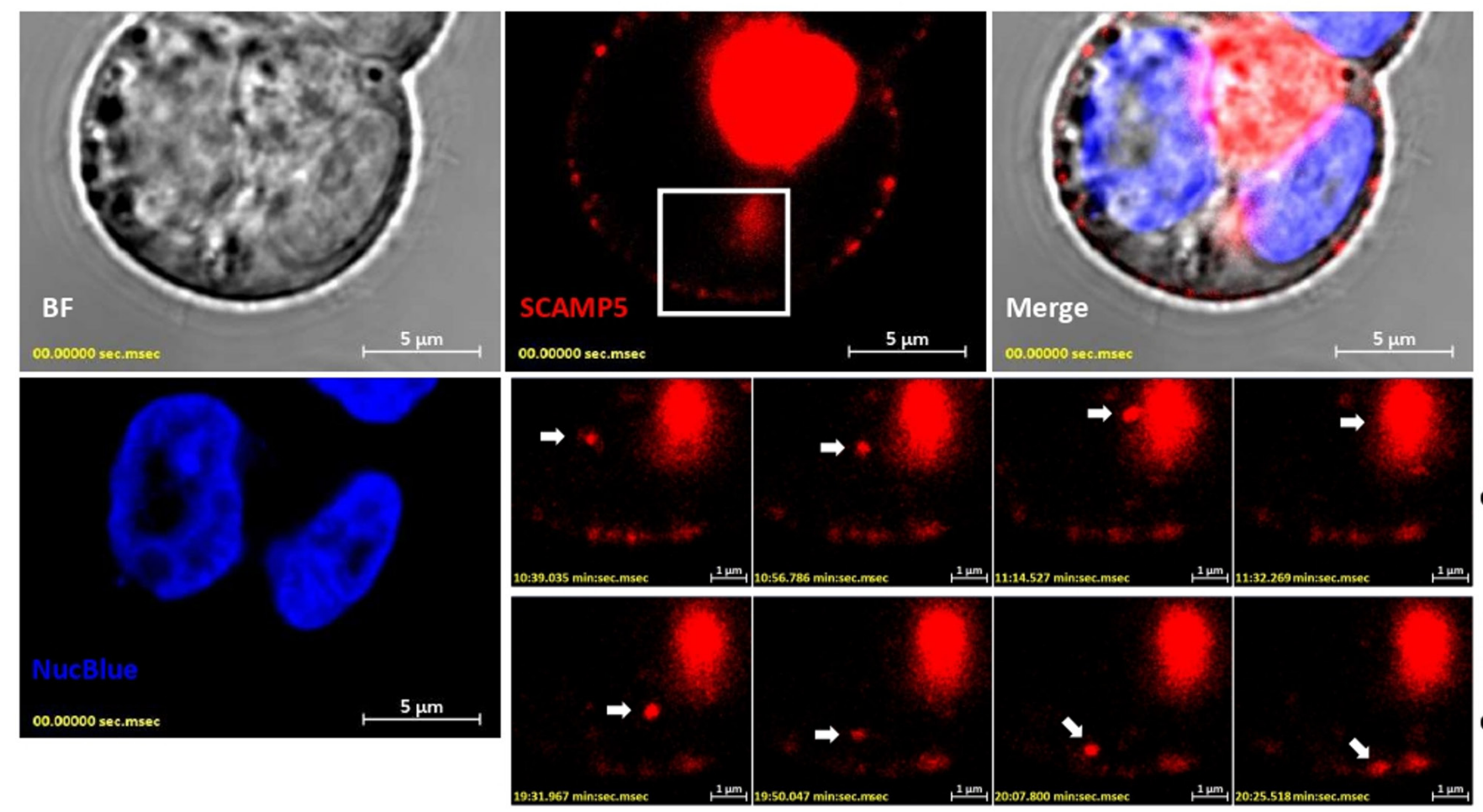

Figure 2 Live cell imaging of SCAMP5 intracellular trafficking. HEK cells transduced with a lentiviral vector to express SCAMP5-mKate2 were imaged by time-lapse confocal microscopy. SCAMP5 was predominantly localised to the Golgi apparatus. Additional SCAMP5 puncta were present at the cell periphery (similar distribution to that observed in figure 1C). Intracellular vesicles bearing SCAMP5 were observed to traffic in both anterograde and retrograde directions between the cell surface and the Golgi (lower right images representing the magnified region indicated by the white box, white arrows trace a unique vesicle across acquisition intervals in the retrograde (upper panels) and anterograde (lower panels) trajectories). See online supplemental materials online supplemental files for SCAMP5-mKate2 construct design. Correlate with online supplemental video 1 , online supplemental video 2, online supplemental video 3, online supplemental video 4. 63x magnification with oil immersion. NucBlue delineates the nucleus. BF, bright-field; HEK, human embryonic kidney.

into human embryonic kidney (HEK) cells. SCAMP5mKate2 localised mainly to the Golgi body, with puncta observed at the cell periphery (figure 2). This distribution matched the SCAMP5 localisation patterns observed by immunofluorescence on endogenously expressed SCAMP5 in primary pDCs (figure 1C). On time-series imaging, SCAMP5 was highly motile in the cell, with both anterograde and retrograde trajectories observed between the Golgi and the plasma membrane (figure 2 and online supplemental video 1 , online supplemental video 2, online supplemental video 3, online supplemental video 4 ).

To probe for a possible interaction between SCAMP5 and the IFN- $\alpha$ trafficking pathway, the fluorescent protein mVenus was generated as a soluble expression construct, designated 'cytosolic/c-mVenus' or with the signal peptide from human IFN- $\alpha$ at the N-terminus (designated 'IFNspmVenus'). Both c-mVenus and IFNsp-mVenus were cloned downstream of a doxycycline-inducible promoter. Expression of c-mVenus in HEK cells revealed a fluorescence pattern that was diffusely present in the cytoplasm, as expected, including in lamellipodia. IFNsp-mVenus displayed a more restricted fluorescence pattern, highlighting the endoplasmic reticulum (ER) throughout the cell (figure 3A and online supplemental video 5, online supplemental video 6). Both c-mVenus and IFNspmVenus were induced in a doxycycline dose-responsive manner. IFNsp-mVenus was detected in the culture media and cell lysates, whereas expression of c-mVenus was only detectable in cell lysates (figure $3 \mathrm{~B}$ ), demonstrating that IFNsp-mVenus was actively secreted. Coexpression of IFNsp-mVenus with SCAMP5-mKate2 demonstrates abundant colocalisation of SCAMP5 and IFNsp-mVenus within the Golgi (figure 3C and online supplemental video 7). Moreover, IFNsp-mVenus, SCAMP5 and the clathrin light chain could be observed within the same vesicle (figure 3D). As clathrin is involved in both endocytic and exocytic pathways, ${ }^{18}$ we hypothesised that SCAMP5 is an integral component of the trafficking machinery in cells in which it is expressed and is positioned to participate in IFN-I secretion mechanisms.

\section{Quantitative expression analysis on SCAMP5 in pDCs from genotyped donors and assays of pDC IFN-I output}

We next sought to investigate the effect SCAMP5 genotypes may have on SCAMP5 expression in pDCs and to determine a potential impact on pDC function. To this end, we obtained fresh blood from homozygous carriers of the risk haplotype and non-risk haplotype tagged by rs2289583 from the Genotype 
A
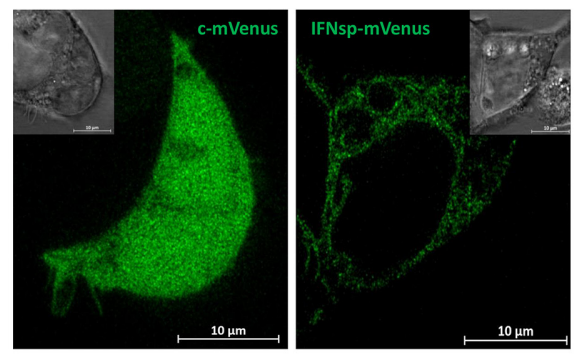

C
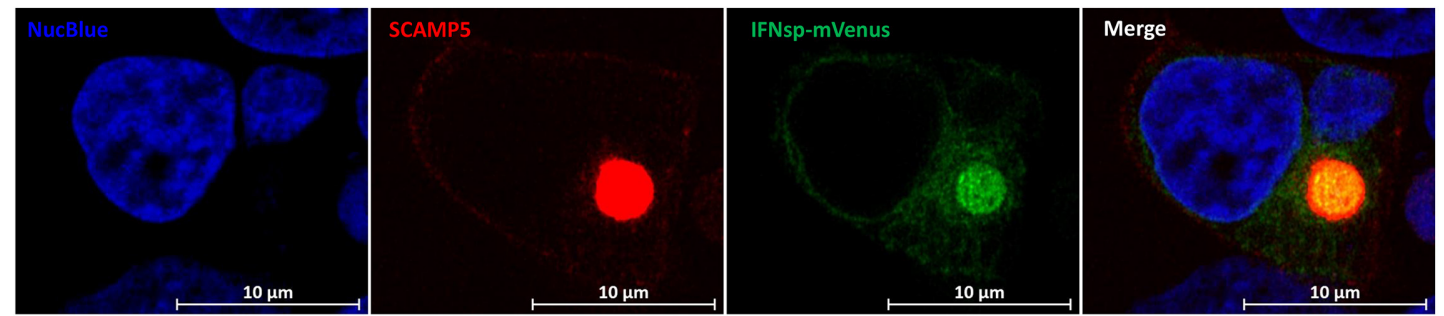

D
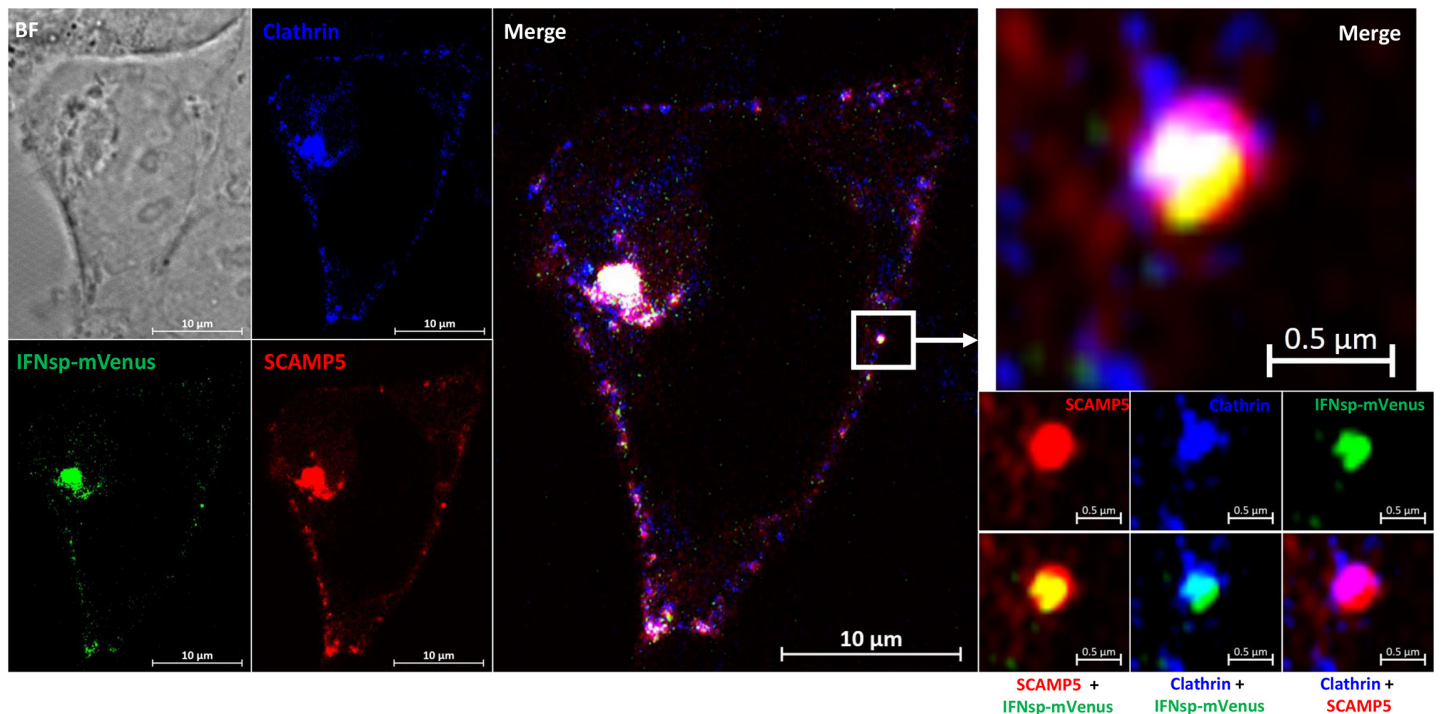

Figure 3 SCAMP5 co-traffics with the IFN- $\alpha$ secretory pathway. Soluble c-mVenus and IFNsp-mVenus were transduced into HEK cells under the regulation of a Tet-On system. (A) Induction using doxycycline revealed that c-mVenus was diffusely expressed in the cytoplasm, whereas IFNsp-mVenus exhibited a lacy pattern characteristic of the ER. Insets represent brightfield images. (B) c-mVenus and IFNsp-mVenus were expressed in a doxycycline dose-responsive manner as determined by western blotting using an anti-GFP antibody. c-mVenus was restricted to intracellular expression, whereas IFNsp-mVenus was detected in cell lysates as well as the culture media. (C) Coexpression of IFNsp-mVenus and SCAMP5-mKate2 reveals colocalisation of SCAMP5 with the IFN- $\alpha$ secretory pathway. (D) SCAMP5 colocalises with IFNsp-mVenus and clathrin in the Golgi apparatus as well as in the vesicles on the cell surface. Magnification of a single vesicle (white box) reveals SCAMP5 and clathrin coating the surface of the vesicle, with IFNsp-mVenus occupying the lumen. (A and C) 63x with oil immersion; (D) 100x with oil immersion. NucBlue delineates the nucleus. See online supplemental material for fluorescent protein designs. Correlate with online supplemental video 5, online supplemental video 6, online supplemental video 7. BF, brightfield; ER, endoplasmic reticulum; GFP, green-fluorescent protein; HEK, human embryonic kidney; IFN, interferon.

and Phenotype Registry, a resource previously used in genotype-phenotype investigations in autoimmune disease (online supplemental tables S1 and S2). ${ }^{19-21}$

For expression quantitative trait locus (eQTL) analysis, quantitative RT-PCR for SCAMP5 and the neighbouring genes, CSK, COX5A, RPP25 and PPCDC, was performed. No significant differences in mRNA levels were seen for SCAMP5, COX5A, RPP25 or PPCDC (figure 4A and online supplemental figure S3). Nominal evidence of an eQTL effect was observed for $C S K$, with elevated CSK transcript in the risk haplotype when normalised to $18 \mathrm{~s}$ rRNA or IRF7 (online supplemental figure S3). To explore whether the risk haplotype polymorphisms might exert their effect at the level of translation, SCAMP5 protein was measured in pDC lysates. Median SCAMP5 protein levels were 
A

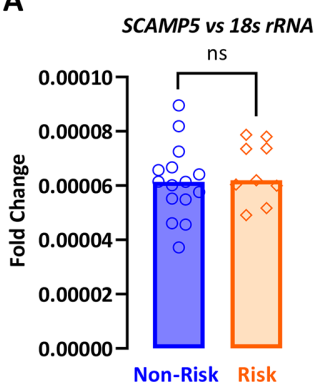

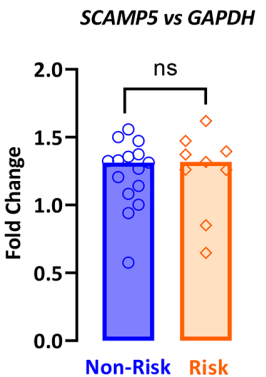

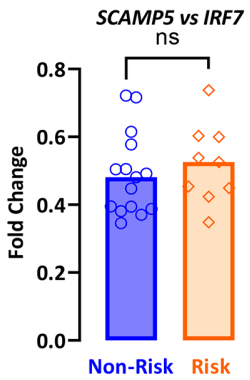

B
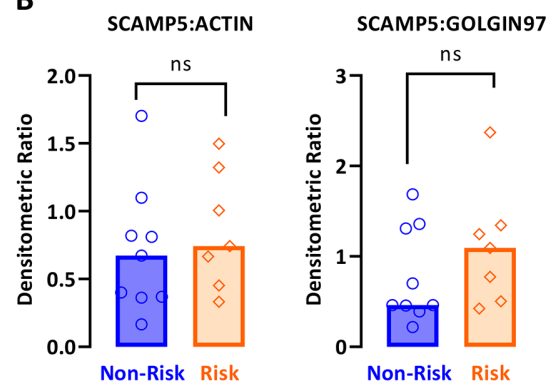

Figure 4 Comparative SCAMP5 expression in pDCs from donors homozygous for the risk or non-risk haplotypes about rs2289583. (A) Quantitative RT-PCR for SCAMP5 transcript from pDCs normalised individually to a highly expressed housekeeping gene (18s rRNA), a moderately expressed housekeeping gene (GAPDH) and a pDC-specific housekeeping gene (IRF7, to account for small differences in pDC purity). (B) Quantitative determination of SCAMP5 protein-level expression by western blotting normalising individually to either actin or golgin-97. Comparisons between genotypes by Mann-Whitney test. ns, not significant; pDCs, plasmacytoid dendritic cells; RT-PCR, reverse transcription PCR.

increased in the risk haplotype when normalised to golgin-97; however, this observation was not statistically significant (figure 4B).

As some risk variants only manifest endophenotypic effects upon cell stimulation (response eQTL, reQTL), ${ }^{22}$ we next assessed for a phenotypic difference between genotypes at the functional level. Culture media from pDCs stimulated with influenza H1N1, imiquimod or ODN-2216 for 4 and 24 hours were assayed for pan-subtype IFN- $\alpha$. When comparing total results from donors for either haplotype, IFN output trended towards lower values for pDCs from the risk group (figure 5A). This difference reached statistical significance only for influenza virus stimulation at the earlier time point.

To determine whether the expression of SCAMP5 in pDCs was influenced by stimulation with influenza virus in a genotype-dependent manner, potentially explaining the kinetic differences in IFN- $\alpha$ production seen above, we assayed for SCAMP5 expression and observed that $S C A M P 5$ is downregulated on stimulation with influenza virus by 4 hours, with no significant differences by genotype (figure 5B). Moreover, there were no consistent differences in IFN- $\alpha$ transcript induction in this condition between the genotypes (figure 5B).

Taken together, these findings suggest, but do not prove, that the $S C A M P 5$ risk haplotype may be related to a delay in IFN protein production or secretion in the early period after viral infection.

\section{Identification of novel SCAMP5 splice variants in pDCs}

To address the possibility that the $S C A M P 5$ risk haplotype influences pDC-specific SCAMP5 isoform expression not
A

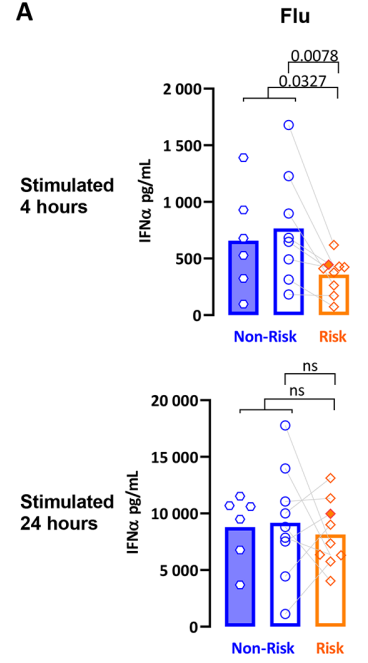

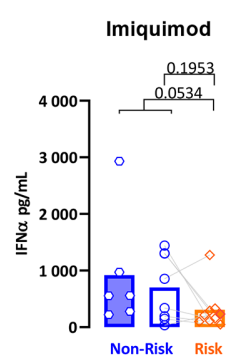
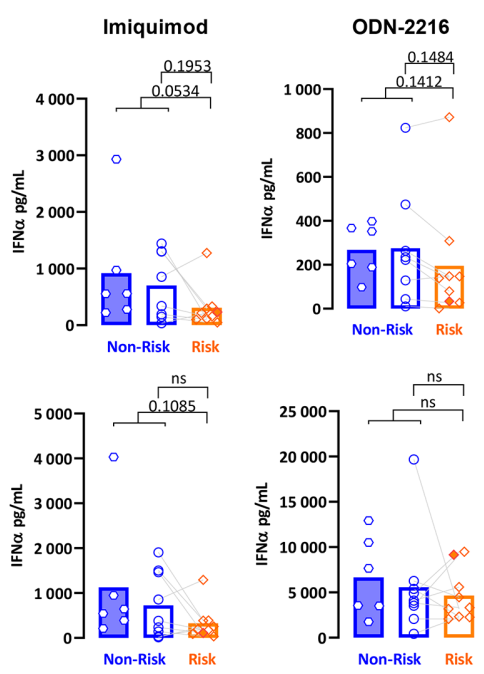

B
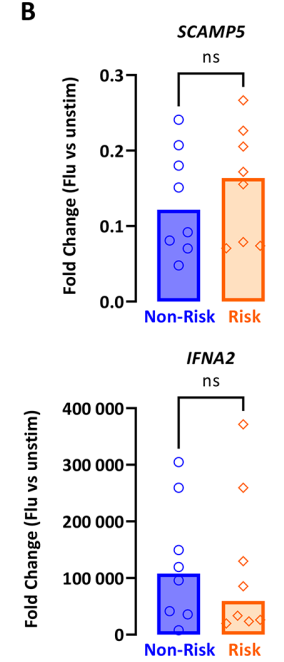
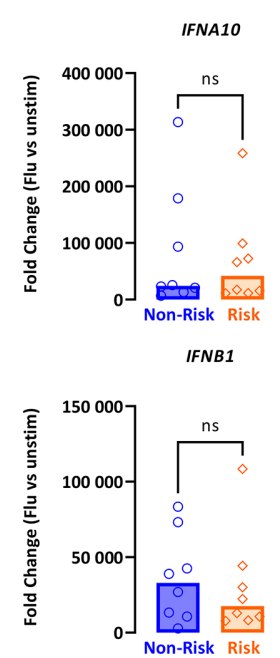

Figure 5 Comparative regulation of IFN- $\alpha$ and SCAMP5 in pDCs from donors homozygous for the risk or non-risk haplotypes about rs2289583 in response to various stimuli. (A) Isolated pDCs were stimulated individually using influenza virus H1N1, imiquimod or ODN-2216 for 4 and 24 hours on separate stimulation plates per time point. Culture media were assayed for IFN- $\alpha$ using a pan-subtype ELISA. Each data point represents biological triplicates of $3000 \mathrm{pDCs}$ per condition. Lines connect paired non-risk/risk pDCs collected and assayed on the same day. (B) Quantitative RT-PCR for SCAMP5, IFNA2, IFNA10 and IFNB1 from pDCs cultured for 4 hours with influenza H1N1. Data were normalised to GUSB expression and compared with paired samples from unstimulated (unstim) wells at 4 hours by the $\triangle \triangle$ Ct method. IFN, interferon; ns, not significant; pDCs, plasmacytoid dendritic cells; RT-PCR, reverse transcription PCR. 
detected by our PCR and protein assays, we recalled an additional four risk donors and five non-risk donors for pDC isolation towards total RNAseq and de novo characterisation of SCAMP5 splice variants. Our data reveal the existence of three novel, intron-retaining SCAMP5 isoforms (online supplemental figure $\mathrm{S} 4$ ). These isoforms were not detected in the human neuroblastoma cell line SH-SY5Y, suggesting pDC-specific expression of these isoforms. Nonetheless, expression of these isoforms did not differ by genotype when specific primers were used for their quantification by quantitative RT-PCR.

\section{DISCUSSION}

We have investigated a novel candidate SLE risk gene, SCAMP5, located on chromosome 15q24. An intronic variant in CSK, $\sim 155 \mathrm{~kb}$ distant from SCAMP5, was previously reported to be associated with SLE by our group and others. ${ }^{11} 16$ However, as shown in figure 1, a more detailed analysis of this region in a larger data set has suggested the presence of an independent genetic effect located within the SCAMP5 gene.

SCAMP5 is a member of the SCAMP gene family and is specifically expressed in neuronal cells of the central nervous system. Interestingly, SCAMP5 is present in the neurohypophysis, derived from neuroectoderm, and is absent in the adenohypophysis, derived from Rathke's pouch, implying tight ontogenic control over SCAMP5 expression. ${ }^{13}$ In cells of the immune system, we found that SCAMP5 is uniquely and highly expressed in pDCs, a rare cell type with the capacity to produce large amounts of IFN-I. Given the importance of IFN-I in SLE, and the large body of literature attributing pDCs as a major source of IFN-I in SLE, we pursued further analysis of SCAMP5 function in pDCs.

SCAMP5 is highly conserved. Rare copy number variations in SCAMP5 can lead to severe neurological phenotypes in humans and mice, while coding-level variants in SCAMP5 are extremely rare. ${ }^{23-26}$ Therefore, we expected that a pDC-specific regulatory variant acting in pDCs underlies the association with SLE. In neuronal cells, the SCAMP family of proteins has been implicated in various roles in intracellular vesicular trafficking, including cargo sorting, endocytosis and exocytosis. ${ }^{27}$ SCAMP5 was shown to be necessary for the sustained release of neurotransmitter-preloaded synaptic vesicles in response to tonic stimulation. In the absence of SCAMP5, membrane recycling pathways were disrupted, leading to the depletion of the synaptic vesicle reserve pool and neurons refractory to further stimulation. ${ }^{28}$ Elsewhere, SCAMP5 has been reported to facilitate the export of signal-peptide containing cytokines, whereas cytokines exported via Golgi-independent pathways were unaffected by SCAMP5 expression. ${ }^{29}$ Taken together, a highly specialised role for SCAMP5 in protein trafficking/ membrane recycling seems likely.

pDCs are noteworthy for their capacity to secrete tremendous amounts of protein, including all members of the IFN-I family (with the exception of IFN-א). This undoubtedly requires efficient mechanisms for protein packaging and subsequent export. IFN-I is secreted along the classical pathway: a signal sequence directs nascent polypeptide chains to the ER, where the protein is translated directly into the ER lumen. Proteins destined for export are then transported to the Golgi apparatus, where sequential protein processing and sorting take place en route to the cell surface via protein-loaded vesicles. ${ }^{30}$ Using IFNsp-mVenus as a traceable surrogate for IFN-I secretion, we have observed that the signal peptide from IFN- $\alpha$ indeed directs protein synthesis to the ER, with later presence in the Golgi. We have also observed that SCAMP5 localises predominantly to the Golgi, with puncta present on the cell surface which traffic to the Golgi in a bidirectional fashion. With these observations, we hypothesised that SCAMP5 licenses the hypersecretory capacity of pDCs by continuous replenishment of Golgi membrane lost to exocytosis on the cell surface. While it stands to reason that any protein destined for secretion via the Golgi may be affected by this process, we focused on IFN- $\alpha$ secondary to its association with SLE and its relative abundance versus other $\mathrm{pDC}$-derived cytokines.

To test our hypotheses, we isolated pDCs to high purity from female donors of childbearing age homozygous for risk or non-risk haplotypes about rs2289583. pDCs from these donors were used in expression-level and functional assays. SCAMP5 expression was similar between the genotypes in unperturbed pDCs, ruling out a baseline eQTL. At the protein level, SCAMP5 had a median higher expression in the risk group when normalised to a Golgi membrane protein loading control; however, the magnitude of this difference and the limited number of samples precluded statistical significance. The possibility that SCAMP5 is differentially regulated between the genotypes upon stimulation was not supported by our reQTL analysis. Finally, whether a novel SCAMP5 isoform exists in pDCs and explains the association with SLE was explored using RNAseq. Novel putative isoforms of SCAMP5 in pDCs were detected; however, they did not differ in expression levels between the genotypes.

The expression of neighbouring genes to either side of $S C A M P 5$ was likewise similar, with the exception of CSK, which exhibits a minor $(<1.2$-fold) yet statistically significant increase in expression in the risk group. This may reflect the small residual LD between rs2289583 and the previously identified independent CSK risk haplotype, which has been associated with increased CSK expression in B cells. However, we note that CSK expression in pDCs is low in comparison with B cells. Thus, to the extent that we can identify pDC phenotypes regulated by rs2289583, $S C A M P 5$ remained a leading candidate gene to explore.

Functionally, pDCs from risk donors produce less IFN- $\alpha$ on average compared with non-risk, when stimulated with imiquimod, ODN-2216 or influenza virus at 4 or 24 hours of stimulation. These differences were more pronounced with imiquimod and influenza virus-both TLR7 agonists-and at the earlier time point. These data 
run counter to the expectation that SLE risk variants in the IFN pathway increase IFN production. With the exception of SLE variants in STAT4 which increase sensitivity to signalling through the IFN- $\alpha / \beta$ receptor, resulting in decreased serum IFN activity, ${ }^{31}$ most IFN-related SLE risk variants indeed confer a greater predilection for IFN production. ${ }^{32}$ Statistically significant differences in these studies usually involve dozens or hundreds of representatives of each genotype, and IFN presence is often inferred through biological activity assays rather than direct IFN measurements as performed here.

Our observations raise several possibilities. The IFN production defect is more pronounced at 4 hours than at 24 hours, suggesting that a kinetic trait in IFN export may be at play. Rapid production of IFN is critical for antiviral defence. As viral prodromes often precede onset of SLE symptoms, it may be the case that enhanced susceptibility to viral infection through slower IFN secretion may induce SLE in an otherwise susceptible host. Alternatively, the observed IFN secretion defect may represent an incidental finding unrelated to SCAMP5's role in pDCs. That SCAMP5 colocalises with the IFN- $\alpha$ secretory pathway does not necessarily indicate an interaction between the two. CRISPR knockout studies in primary pDCs may be necessary for establishing a definitive link between SCAMP5 and IFN secretion. We have successfully used CRISPR to knock out BDCA4 expression in primary human pDCs (online supplemental figure S5); however, knockout of SCAMP5 has proven elusive, possibly due to longer half-life of this protein in these short-lived cells.

Separately, we have investigated human pDC responses to influenza virus by secretome profiling using liquid chromatography tandem mass spectrometry and at the single pDC level using single cell RNAseq (Ghanem MH et $a l$, 2021, manuscript in preparation). There, we observed that SCAMP5 is widely expressed in pDCs immediately ex vivo, but is downregulated upon stimulation, implying a role of SCAMP5 that is distinct from ongoing IFN export. Moreover, we observed evidence for granzyme B release from these cells via a mechanism consistent with degranulation rather than the classic secretory pathway. A role of SCAMP5 in this process would bear more similarity to synaptic vesicle release from neuronal cells where the function of SCAMP5 is more thoroughly described. These possibilities are being addressed by ongoing investigation. This work marks the first description of a role of SCAMP5 in $\mathrm{pDC}$ biology and IFN secretion; much remains to be explored.

Acknowledgements We would like to acknowledge the participants of the GaP Registry for their contribution to this work. Gila Klein, Margaret DeFranco, Kristine Elmaliki and Dorean Flores coordinated GaP donor recall and sample collections. Professor Timothy Vyse (King's College London) kindly provided primary SNP data on SLE cases and controls. Christopher Colon, Heriberto Borrero and Barrett Waling provided assistance with flow cytometry. Amanda Chan and Anita Ng provided assistance with confocal microscopy. Anthony Liew performed the library construction and sequencing for total RNAseq. We thank Professor David Castle (University of Virginia) for helpful discussions on SCAMP5. Lentiviral constructs were cloned using vectors provided by Addgene.
Contributors MHG, SJK, KRS and PKG conceived and designed the study. MHG, HV and LNC collected the experimental data. MHG and KRS analysed the experimental data. AS performed the RNAseq analysis. WL performed the statistical genetics analysis. MHG, KRS and PKG wrote the manuscript. PKG was the principal investigator on the study and serves as the guarantor of this work. All authors revised and approved the final manuscript.

Funding This work is supported by a career development award to KRS (NIH K01AR071502) and awards to SJK (R01 AR075565 and U19 Al144306), as well as departmental funds provided by the Center for Genomics and Human Genetics at the Feinstein Institutes for Medical Research.

Competing interests None declared.

Patient consent for publication Not required.

Ethics approval The Genotype and Phenotype (GaP) Registry at the Feinstein Institutes for Medical Research provided de-identified blood from donors consented under an IRB-approved protocol (IRB\# 09-081). The Committee for Participant Protection at the Feinstein Institutes for Medical Research approved this study (TAP0307.5.73). The GaP is a subprotocol of the Tissue Donation Program (TDP) at Northwell Health and a national resource for genotype-phenotype studies (https:// www.feinsteininstitute.org/robert-sboas-center-for-genomics-and-human-genetics/ gap-registry/).

Provenance and peer review Not commissioned; externally peer reviewed.

Data availability statement Data are available in a public, open access repository. Data are available upon reasonable request. RNA-seq data will be uploaded to GEO and may also be directly available through our group.

Supplemental material This content has been supplied by the author(s). It has not been vetted by BMJ Publishing Group Limited (BMJ) and may not have been peer-reviewed. Any opinions or recommendations discussed are solely those of the author(s) and are not endorsed by BMJ. BMJ disclaims all liability and responsibility arising from any reliance placed on the content. Where the content includes any translated material, BMJ does not warrant the accuracy and reliability of the translations (including but not limited to local regulations, clinical guidelines, terminology, drug names and drug dosages), and is not responsible for any error and/or omissions arising from translation and adaptation or otherwise.

Open access This is an open access article distributed in accordance with the Creative Commons Attribution Non Commercial (CC BY-NC 4.0) license, which permits others to distribute, remix, adapt, build upon this work non-commercially, and license their derivative works on different terms, provided the original work is properly cited, appropriate credit is given, any changes made indicated, and the use is non-commercial. See: http://creativecommons.org/licenses/by-nc/4.0/.

\section{ORCID iDs}

Mustafa H Ghanem http://orcid.org/0000-0002-8019-5324

Andrew J Shih http://orcid.org/0000-0001-8375-7313

Himanshu Vashistha http://orcid.org/0000-0003-4653-5011

Latanya N Coke http://orcid.org/0000-0001-9018-0188

Wentian Li http://orcid.org/0000-0003-1155-110X

Sun Jung Kim http://orcid.org/0000-0001-8535-5537

Kim R Simpfendorfer http://orcid.org/0000-0002-1906-2899

Peter K Gregersen http://orcid.org/0000-0003-1613-1518

\section{REFERENCES}

1 Reizis B. Plasmacytoid dendritic cells: development, regulation, and function. Immunity 2019;50:37-50.

2 Baechler EC, Batliwalla FM, Karypis G, et al. Interferon-Inducible gene expression signature in peripheral blood cells of patients with severe lupus. Proc Natl Acad Sci U S A 2003;100:2610-5.

3 Vermi W, Lonardi S, Morassi M, et al. Cutaneous distribution of plasmacytoid dendritic cells in lupus erythematosus. Selective tropism at the site of epithelial apoptotic damage. Immunobiology 2009;214:877-86.

4 Arazi A, Rao DA, Berthier CC, et al. The immune cell landscape in kidneys of patients with lupus nephritis. Nat Immunol 2019;20:902-14.

5 Furie R, Werth VP, Merola JF, et al. Monoclonal antibody targeting BDCA2 ameliorates skin lesions in systemic lupus erythematosus. $J$ Clin Invest 2019;129:1359-71.

6 Sisirak V, Ganguly D, Lewis KL, et al. Genetic evidence for the role of plasmacytoid dendritic cells in systemic lupus erythematosus. J Exp Med 2014;211:1969-76. 
7 Son M, Kim SJ, Diamond B. SLE-associated risk factors affect DC function. Immunol Rev 2016;269:100-17.

8 Jego G, Palucka AK, Blanck J-P, et al. Plasmacytoid dendritic cells induce plasma cell differentiation through type I interferon and interleukin 6. Immunity 2003;19:225-34.

9 Soni C, Perez OA, Voss WN, et al. Plasmacytoid dendritic cells and type I interferon promote extrafollicular B cell responses to extracellular Self-DNA. Immunity 2020;52:1022-38.

10 Crow MK, Olferiev M, Kirou KA. Type I interferons in autoimmune disease. Annu Rev Pathol 2019;14:369-93.

11 Bentham J, Morris DL, Graham DSC, et al. Genetic association analyses implicate aberrant regulation of innate and adaptive immunity genes in the pathogenesis of systemic lupus erythematosus. Nat Genet 2015;47:1457-64.

12 Hubbard C, Singleton D, Rauch M, et al. The secretory carrier membrane protein family: structure and membrane topology. Mol Biol Cell 2000;11:2933-47.

13 Fernández-Chacón R, Südhof TC. Novel SCAMPs lacking NPF repeats: ubiquitous and synaptic vesicle-specific forms implicate SCAMPs in multiple membrane-trafficking functions. J Neurosci 2000;20:7941-50.

14 Uhlén M, Fagerberg L, Hallström BM, et al. Proteomics. Tissuebased map of the human proteome. Science 2015;347:1260419.

15 Hom G, Graham RR, Modrek B, et al. Association of Systemic Lupus Erythematosus with C8orf13-BLK and ITGAM-ITGAX. N Engl J Med Overseas Ed 2008;358:900-9.

16 Manjarrez-Orduño N, Marasco E, Chung SA, et al. Csk regulatory polymorphism is associated with systemic lupus erythematosus and influences B-cell signaling and activation. Nat Genet 2012;44:1227-30.

17 Su Al, Cooke MP, Ching KA, et al. Large-Scale analysis of the human and mouse transcriptomes. Proc Natl Acad Sci U S A 2002;99:4465-70.

18 Brodsky FM. Diversity of clathrin function: new tricks for an old protein. Annu Rev Cell Dev Biol 2012;28:309-36.

19 Simpfendorfer KR, Armstead BE, Shih A, et al. Autoimmune diseaseassociated haplotypes of blk exhibit lowered thresholds for B cell activation and expansion of Ig class-switched B cells. Arthritis Rheumatol 2015;67:2866-76.

20 Li D, Matta B, Song S, et al. IRF5 genetic risk variants drive myeloidspecific IRF5 hyperactivation and presymptomatic SLE. JCI Insight
2020;5. doi:10.1172/jci.insight.124020. [Epub ahead of print: $30 \mathrm{Jan}$ 2020].

21 Gregersen PK, Klein G, Keogh M, et al. The genotype and phenotype (GAP) registry: a living Biobank for the analysis of quantitative traits. Immunol Res 2015;63:107-12.

22 Kim-Hellmuth S, Bechheim M, Pütz B, et al. Genetic regulatory effects modified by immune activation contribute to autoimmune disease associations. Nat Commun 2017;8:266.

23 Castermans D, Volders K, Crepel A, et al. SCAMP5, NBEA and AMISYN: three candidate genes for autism involved in secretion of large dense-core vesicles. Hum Mol Genet 2010;19:1368-78.

24 Hubert L, Cannata Serio M, Villoing-Gaudé L, et al. De novo SCAMP5 mutation causes a neurodevelopmental disorder with autistic features and seizures. J Med Genet 2020;57:138-44.

25 Zhang D, Yuan C, Liu M, et al. Deficiency of SCAMP5 leads to pediatric epilepsy and dysregulation of neurotransmitter release in the brain. Hum Genet 2020;139:545-55.

26 Dickinson ME, Flenniken AM, Ji X, et al. High-throughput discovery of novel developmental phenotypes. Nature 2016;537:508-14.

27 Law AHY, Chow C-M, Jiang L. Secretory carrier membrane proteins. Protoplasma 2012;249:269-83.

28 Park D, Lee U, Cho E, et al. Impairment of release site clearance within the active zone by reduced SCAMP5 expression causes short-term depression of synaptic release. Cell Rep 2018;22:3339-50.

29 Han C, Chen T, Yang M, et al. Human SCAMP5, a novel secretory carrier membrane protein, facilitates calcium-triggered cytokine secretion by interaction with SNARE machinery. J Immunol 2009;182:2986-96.

30 Lacy P, Stow JL. Cytokine release from innate immune cells: association with diverse membrane trafficking pathways. Blood 2011;118:9-18

31 Kariuki SN, Kirou KA, MacDermott EJ, et al. Cutting edge: autoimmune disease risk variant of STAT4 confers increased sensitivity to IFN-alpha in lupus patients in vivo. $\mathrm{J}$ Immunol 2009;182:34-8.

32 Ghodke-Puranik Y, Niewold TB. Genetics of the type I interferon pathway in systemic lupus erythematosus. Int J Clin Rheumtol 2013;8:657-69. 